\title{
Covariant generalization of the ISGW quark model
}

\author{
D. Tadić and S. Žganec \\ Physics Department, University of Zagreb, Bijenička c. 32, Zagreb, Croatia
}

\begin{abstract}
A fairly general Lorentz-covariant quark model of mesons is constructed. It has several versions whose nonrelativistic limit corresponds to the well-known Isgur, Scora, Grinstein, and Wise model. In the heavy-quark limit, the covariant model naturally and automatically produces the heavy-quark symmetry results for meson decay constants and semileptonic decay form factors. The meson decay constants and the Isgur-Wise functions are calculated for various versions of the covariant model and compared with other estimates. A general and adaptable structure of the covariant model ensures that it can be used to describe transitions involving light and/or heavy mesons.
\end{abstract}




\section{Introduction}

The well-established, simple, and often used, nonrelativistic quark model of Isgur, Scora, Grinstein, and Wise (ISGW) [1] has also been employed $[2,3,4,5]$ in the investigations of heavy-quark symmetry (HQS). Although the ISGW model helped in HQS investigations, this nonrelativistic model was not capable $[4,5]$ of properly reproducing all of the heavy-quark effective-theory (HQET) relations among semileptonic meson decay form factors. It had to be "relativized" to some extent [2,4,5]. Moreover, even in the original paper [1] some compensation for relativistic effects had to be introduced with meson wave functions. In this way, useful insights in the HQET were gained and subleading corrections of order $\bar{\Lambda} / m_{Q}$ were estimated [5] (Here $\bar{\Lambda} \sim \Lambda_{Q C D}$ and $m_{Q}$ is the heavy-quark mass).

Thus it seemed useful to develop a fully covariant model that, in the nonrelativistic limit (NRL), goes into the ISGW model. It turned out that such a covariant model can, to a great extent, retain the simplicity, which was an endearing and useful feature of the nonrelativistic model [1].

The covariant model can have a fairly general form [6] that can be, if wanted, specified in such a way as to lead to the ISGW model in the NRL. The given covariant formulation allows reasonable freedom in the selection of model parameters and model meson wave functions. They can be selected to reproduce a particular Isgur-Wise function (IWF), what might provide a good basis for the calculation of $\bar{\Lambda} / m_{Q}$ corrections.

An important feature in all variations of the proposed covariant quark model (CQM) is the description of valence quarks (antiquarks). They are parametrized by the onmass-shell Dirac spinors, as it was the case in earlier models [1] and in all subsequent usages [2-6]. In a covariant model, such a description might lead to difficulties with 
the covariant definition of a meson mass $M$. As shown in the next section, this can be resolved by introducing a scalar function that represents the neutral sea with a momentum $K$ and vacuum quantum numbers [6]. This is an attractive feature, as sea contributions must figure in a description of a hadron. In the present model, which takes into account only fluctuations involving valence quarks (antiquarks), the sea is described in the simplest possible way, as a physical vacuum. The sea momentum function $F(K)$ has a particularly simple form if one wants to define a model that in the NRL goes into the ISGW model.

In the third section of this paper the meson decay constants and the IWF are calculated for this version of the CQM inspired by the ISGW model. It turns out that the covariant formulation takes care of the relativistic effects, which previously had to be compensated for by a phenomenological parameter $\kappa$ (see Fig. 1). The sea function in this version of the CQM is just a Dirac delta function, which ensures that a meson has a properly defined on-mass-shell four-momentum $P,\left(P^{2}=M^{2}\right)$.

However, one could use a nontrivial $F(K)$ function in the CQM. The form of such a function would influence the model description of the physical quantities. This is illustrated in the concluding sections of this paper by calculating meson decay constants and the IWF for a Gaussian $F(K)$. 


\section{Relativistic model(s) and the ISGW limit}

A meson $H$ with the four-momentum $P$ and the mass $M$ is covariantly represented by

$$
\begin{gathered}
|H(E, \vec{P}, M)\rangle=N \sum_{c, s_{1}, s_{2}} \int\left[4 m_{Q} m_{d}\right] d^{4} p \delta^{4}\left(p^{2}-m_{Q}^{2}\right) \Theta(e) \\
\cdot d^{4} q \delta\left(q^{2}-m_{d}^{2}\right) \Theta(\epsilon) d^{4} K F(K) \delta^{(4)}(p+q+K-P) \Theta(E) \phi\left(l_{\perp}\right) \\
\cdot \bar{u}_{Q, s_{1}}^{c}(\vec{p}) \gamma_{5} v_{d, s_{2}}^{c}(\vec{q}) d_{d}^{+}\left(\vec{q}, c, s_{2}\right) b_{Q}^{+}\left(\vec{p}, c, s_{1}\right)|0\rangle
\end{gathered}
$$

Here, the index $d$ refers for concreteness, to a light $d$ antiquark, whereas the index $Q$ denotes any of heavy quarks. The Dirac functions such as $\delta\left(p^{2}-m_{Q}^{2}\right)$, combined with the corresponding step function $\Theta(e)$, ensure that valence quarks are on the mass shell. This is a characteristic feature of the ISGW model. One can select, if desired, a quark wave function $\phi\left(l_{\perp}\right)$ which goes into the ISGW wave function in the NRL. Various momenta in $(2.1)$ are

$$
\begin{gathered}
l_{\perp}^{\mu}(P)=l^{\mu}-\frac{P^{\mu}(P \cdot l)}{M^{2}} \\
l^{\mu}=\frac{1}{2}\left(p^{\mu}-q^{\mu}\right) \\
p^{\mu}=(e, \vec{p}) ; q^{\mu}=(\epsilon, \vec{q}) \\
l_{\perp}^{\mu}(M)=\left(0, \frac{\vec{p}-\vec{q}}{2}\right)
\end{gathered}
$$

The ISGW limit is obtained if the wave function is selected as 


$$
\phi\left(l_{\perp}^{\mu}\right)=\frac{1}{\pi^{3 / 4} \beta_{S}^{3 / 2}} e^{+\left(l_{\perp}^{\mu}\right)^{2} / 2 \beta_{S}^{2}}
$$

Here $\beta_{S}$ corresponds to the variational solution [1] with the harmonic-oscillator wave functions.

The sea function $F(K)$ ensures that the meson mass M can be covariantly defined. Without $F(K)$, the Dirac delta function $\delta^{(4)}(p+q-P)$ leads in the rest frame $(\vec{P}=0)$ to the momentum-dependent mass [1] $M=\left(\vec{p}^{2}+m_{Q}^{2}\right)^{1 / 2}+\left(\vec{p}^{2}+m_{d}^{2}\right)^{1 / 2}$. Generally, one has the same freedom in selecting $F(K)$ as one had in selecting $\phi\left(l_{\perp}\right)$. However, the ISGW state vectors of the weak-binding limit (WBL) [1] will be obtained if a simple form is selected:

$$
F(K)=\delta^{(4)}\left[K^{\mu}-\frac{P^{\mu}}{M}\left(\frac{P^{\nu}}{M}(P-(p+q))_{\nu}\right]\right.
$$

In the meson rest frame:

$$
K^{0}(\vec{P}=0)=[M-e-\epsilon]=\mu_{K}(\vec{p}, \vec{q}) \quad ; \quad \vec{K}(\vec{P}=0)=0
$$

Obviously, as $\mu_{K}(\vec{p}, \vec{q})$ is not always positive, the $\mathrm{K}$ does not correspond to a physical, on-mass-shell particle. It can be associated with some sea contribution. This contribution can, in principle, have a less naive form than (2.4), which has been inspired by the ISGW limit. For example, one could try (see Sec. IV) the form

$$
F(K)=\delta^{(4)}\left[K^{\mu}-\frac{P^{\mu}}{M}\left(\frac{P^{\nu}}{M}(P-(p+q))_{\nu}\right] e^{-\alpha K^{2}}\right.
$$

It also leads to the ISGW model in the NRL.

After performing the integrations $d^{4} K, d p^{0}$, and $d q^{0}$ in (2.1), one is left with

$$
\begin{aligned}
\mid H(E, \vec{P}, M)>= & N \sum_{c, s_{1}, s_{2}} \int d^{3} p \frac{m_{Q}}{e} d^{3} q \frac{m_{d}}{\epsilon} \delta^{(4)}\left[(p+q)^{\mu}-\frac{P^{\mu}}{M}\left(\frac{E(e+\epsilon)}{M}-\frac{\vec{P}(\vec{p}+\vec{q})}{M}\right)\right] \\
& \cdot \phi\left(l_{\perp}\right) \bar{u}_{Q, s_{1}}^{c}(\vec{p}) \gamma_{5} v_{d, s_{2}}^{c}(\vec{q}) d_{d}^{+}\left(\vec{q}, c, s_{2}\right) b_{Q}^{+}\left(\vec{p}, c, s_{1}\right) \mid 0>
\end{aligned}
$$


Using the notation

$$
p_{\perp}^{\mu}=p^{\mu}-\frac{P^{\mu}}{M^{2}}(p \cdot P) \quad ; \quad p_{\|}^{\mu}=\frac{P^{\mu}}{M^{2}}(p \cdot P)
$$

one realizes that the Dirac delta function in (2.7) constrains the orthogonal components of the quark four-vectors, i.e.,

$$
p_{\perp}^{\mu}+q_{\perp}^{\mu}=0
$$

In the meson rest frame this gives the ISGW relation

$$
\vec{p}+\vec{q}=0
$$

By rewriting the complex $\delta$ function in (2.7) one obtains a more manageable form

$$
\begin{gathered}
\mid H(E, \vec{P}, M)>=N \sum_{c, s_{1}, s_{2}} \int d^{3} p \frac{m_{Q}}{e} d^{3} q \frac{m_{d}}{\epsilon} \frac{1}{\frac{E^{2}}{M^{2}}\left(1-\frac{\vec{P} \vec{q}}{E \epsilon}\right)} \delta^{(3)}\left(\vec{q}+\vec{p}-\frac{\vec{P}}{M}\left(p_{\|}\right) T\right) \\
\cdot \phi\left(l_{\perp}\right) \bar{u}_{Q, s_{1}}^{c}(\vec{p}) \gamma_{5} v_{d, s_{2}}^{c}(\vec{q}) d_{d}^{+}\left(\vec{q}, c, s_{2}\right) b_{Q}^{+}\left(\vec{p}, c, s_{1}\right) \mid 0>
\end{gathered}
$$

Here the quantities $T$ and $p_{\|}$are

$$
T=1+\frac{\sqrt{m_{d}^{2}-m_{Q}^{2}+p_{\|}^{2}}}{p_{\|}} \quad ; \quad p_{\|}=\frac{P \cdot p}{M}
$$

In the NRL and the WBL [1],

$$
\begin{gathered}
\vec{p}^{2}<<m_{Q}^{2} \quad ; \quad \vec{q}^{2}<<m_{d}^{2} \\
E \rightarrow M \quad ; \quad e \rightarrow m_{Q} \quad ; \quad \epsilon \rightarrow m_{d} \\
p_{\|} \rightarrow m_{Q}-\frac{\vec{P} \vec{p}}{M} \quad ; \quad \vec{q} \rightarrow-\vec{p}+\left(m_{Q}+m_{d}\right) \frac{\vec{P}}{M} \\
\left(l_{\perp}^{\mu}\right)^{2} \rightarrow 2 m_{Q} \frac{\vec{P} \vec{p}}{M}-\vec{p}^{2} \quad ; \quad \bar{u}_{Q, s_{1}}(\vec{p}) \gamma_{5} v_{d, s_{2}}(\vec{q}) \rightarrow \delta_{s_{1},-s_{2}}
\end{gathered}
$$

one finds 


$$
\begin{gathered}
\mid H(E, \vec{P}, M)>_{N R, W B}=N \sum_{c, s_{1}, s_{2}} \int d^{3} p\left[\frac{1}{\pi^{3 / 4} \beta_{S}^{3 / 2}} e^{\left(-\vec{p}^{2}+2 m_{Q} \frac{\vec{P} \vec{p}}{M}\right) /_{2} \beta_{s}^{2}}\right] \\
\cdot \delta_{s_{1}-s_{2}} d_{d}^{+}\left(-\vec{p}+\left(m_{d}+m_{Q}\right) \frac{\vec{P}}{M}, c, s_{2}\right) b_{Q}^{+}\left(\vec{p}, c, s_{1}\right) \mid 0>
\end{gathered}
$$

The substitution

$$
\vec{p}=\overrightarrow{p^{\prime}}+\frac{m_{Q}}{M} \vec{P}
$$

leads to the well-known [1-5] ISGW form

$$
\begin{gathered}
\mid H(E, \vec{P}, M)>_{N R, W B}=N \sum_{c, s_{1}, s_{2}} \int d^{3} p^{\prime}\left[\frac{1}{\pi^{3 / 4} \beta_{S}^{3 / 2}} e^{-{\overrightarrow{p^{\prime}}}^{2} / 2 \beta_{S}^{2}}\right] \\
\cdot \delta_{s_{1},-s_{2}} d_{d}^{+}\left(-\overrightarrow{p^{\prime}}+\frac{m_{d}}{M} \vec{P}, c, s_{2}\right) b_{Q}^{+}\left(\overrightarrow{p^{\prime}}+\frac{m_{Q}}{M} \vec{P}, c, s_{1}\right) \mid 0>
\end{gathered}
$$

The full covariant forms (2.1) or (2.11) lead to fully covariant predictions for meson form factors in the CQM, as shown below. These states can be covariantly normalized. With

$$
<H\left(E^{\prime}, \overrightarrow{P^{\prime}}, M\right) \mid H(E, \vec{P}, M)>=2 E \delta^{(3)}\left(\vec{P}-\vec{P}^{\prime}\right)
$$

one finds for the state (2.7):

$$
\begin{gathered}
(2 \pi)^{6} 3 N(\vec{P})^{2} \int d^{3} p \frac{m_{Q} m_{d}}{e \epsilon}\left[\phi\left(l_{\perp}\right)\right]^{2} \frac{1}{\left[\frac{E^{2}}{M^{2}}\left(1-\frac{\vec{P} \vec{q}}{E \epsilon}\right)\right]^{2}} \frac{1}{\left(\frac{p}{M}\right)^{3}} \\
\left.\cdot \frac{1}{\left[1+\frac{\vec{P}(e \vec{P}-E \vec{p})}{E M \sqrt{m_{d}^{2}-m_{Q}^{2}+p_{\|}^{2}}}\right]} \frac{p \cdot q+m_{Q} m_{d}}{m_{Q} m_{d}} \delta^{(3)}\left(\vec{P}-\vec{P}^{\prime}\right)\right|_{\vec{q}=-\vec{p}+\frac{\vec{P}}{M}\left(p_{\|}\right) T}=2 E \delta^{3}\left(\vec{P}-\vec{P}^{\prime}\right)
\end{gathered}
$$

From this expression, $N(\vec{P})$ is

$$
N(\vec{P})=\frac{E}{M} N(0)
$$

where $N(0)$ can be calculated numerically. 


\section{Meson form factors and the Isgur-Wise function}

The form factors for $B \rightarrow D\left(D^{*}\right)$ semileptonic transitions are defined in the standard way:

$$
\begin{gathered}
<D^{+}\left(\vec{P}_{D}\right)\left|\bar{c} \gamma^{\mu} b\right| \bar{B}^{0}\left(\vec{P}_{B}\right)>=\frac{1}{(2 \pi)^{3}}\left[f_{+}\left(P_{B}+P_{D}\right)^{\mu}+f_{-}\left(P_{B}-P_{D}\right)^{\mu}\right] \\
<D^{*+}\left(\epsilon, \vec{P}_{D}\right)\left|\bar{c} \gamma^{\mu} b\right| \bar{B}^{0}\left(\vec{P}_{B}\right)>=\frac{i}{(2 \pi)^{3}} g \epsilon^{\mu \nu \rho \sigma} \epsilon_{\nu}^{*}\left(P_{B}+P_{D}\right)_{\rho}\left(P_{B}-P_{D}\right)_{\sigma} \\
<D^{*+}\left(\epsilon, \vec{P}_{D}\right)\left|\bar{c} \gamma^{\mu} \gamma_{5} b\right| \bar{B}^{0}\left(\vec{P}_{B}\right)>=\frac{1}{(2 \pi)^{3}}\left[f \epsilon^{* \mu}+a_{+}\left(\epsilon^{*} \cdot P_{B}\right)\left(P_{B}+P_{D}\right)^{\mu}+a_{-}\left(\epsilon^{*} \cdot P_{B}\right)\left(P_{B}-P_{D}\right)^{\mu}\right]
\end{gathered}
$$

Here the vector meson state $\left|D^{*}\right\rangle$ is obtained from (2.1) by replacing $\gamma_{5}$ by $(\epsilon \gamma)$. For example, one finds that

$$
\begin{gathered}
<D^{+}\left(\vec{P}_{D}\right)\left|\bar{c} \gamma^{\mu} b\right| \overrightarrow{B^{0}}\left(\vec{P}_{B}\right)>=3(2 \pi)^{3} N_{D}\left(\vec{P}_{D}\right) N_{B}\left(\vec{P}_{B}\right) \sum_{s_{1}, s_{2}, s_{1}^{\prime}} \int d^{3} p^{\prime} d^{3} p \frac{m_{c} m_{b}}{e^{\prime} e} \frac{m_{d}}{\epsilon^{\prime}} \\
\cdot \frac{1}{\frac{E_{D}^{2}}{M_{D}^{2}}\left(1-\frac{\vec{P}_{D} \overrightarrow{q^{\prime}}}{E_{D} \epsilon^{\prime}}\right)} \frac{1}{\frac{E_{B}^{2}}{M_{B}^{2}}\left(1-\frac{\vec{P}_{B}{\overrightarrow{q^{\prime}}}^{\prime}}{E_{B} \epsilon^{\prime}}\right)} \delta^{(3)}\left[-\vec{p}+\frac{\vec{P}_{B}}{M_{B}}\left(p_{B \|}\right) T_{B}+\overrightarrow{p^{\prime}}-\frac{\vec{P}_{D}}{M_{D}}\left(p_{D \|}\right) T_{D}\right] \\
\cdot \phi_{D}\left(l_{D \perp}\right) \phi_{B}\left(l_{B \perp}\right)\left[-\bar{v}_{d, s_{2}}\left(\overrightarrow{q^{\prime}}\right) \gamma_{5} u_{c, s_{1}^{\prime}}\left(\overrightarrow{p^{\prime}}\right) \bar{u}_{c, s_{1}^{\prime}}\left(\overrightarrow{p^{\prime}}\right) \gamma^{\mu} u_{b, s_{1}}(\vec{p}) \bar{u}_{b, s_{1}}(\vec{p}) \gamma_{5} v_{d, s_{2}}\left(\overrightarrow{q^{\prime}}\right)\right]
\end{gathered}
$$

Here

$$
\begin{array}{cc}
p_{D \|}=\frac{E_{D} e^{\prime}-\vec{P}_{D} \overrightarrow{p^{\prime}}}{M_{D}} & p_{B \|}=\frac{E_{B} e-\vec{P}_{B} \vec{p}}{M_{B}} \\
T_{D}=1+\frac{\sqrt{m_{d}^{2}-m_{c}^{2}+\left(p_{D \|}\right)^{2}}}{p_{D \|}} & T_{B}=1+\frac{\sqrt{m_{d}^{2}-m_{b}^{2}+\left(p_{B \|}\right)^{2}}}{p_{B \|}}
\end{array}
$$




$$
e^{\prime}=\sqrt{m_{c}^{2}+{\overrightarrow{p^{\prime}}}^{2}} \quad e=\sqrt{m_{b}^{2}+\vec{p}^{2}}
$$

In the B-meson rest frame $\left(\vec{P}_{B}=0\right)$ this becomes

$$
\begin{gathered}
<D^{+}\left(\vec{P}_{D}\right)\left|\bar{c} \gamma^{\mu} b\right| \vec{B}^{0}(0)>=3(2 \pi)^{3} N_{D}\left(\vec{P}_{D}\right) N_{B}(0) \int d^{3} p^{\prime} d^{3} p \frac{m_{c} m_{b}}{e^{\prime} e} \frac{m_{d}}{\epsilon^{\prime}} \\
\cdot \frac{1}{\frac{E_{D}^{2}}{M_{D}^{2}}\left(1-\frac{\vec{P}_{D} \vec{q}^{\prime}}{E_{D} \epsilon^{\prime}}\right)} \delta^{(3)}\left(-\vec{p}+\vec{p}^{\prime}-\frac{\vec{P}_{D}}{M_{D}}\left(p_{D \|}\right) T_{D}\right) \phi_{D} \phi_{B} \cdot\left\{V^{\mu}\right\}
\end{gathered}
$$

Here

$$
\begin{gathered}
\left\{V^{\mu}\right\}=\frac{1}{8} \operatorname{Tr}\left[\gamma_{5}\left(1+\frac{\not p^{\prime}}{m_{c}}\right) \gamma^{\mu}\left(1+\frac{\not p}{m_{b}}\right) \gamma_{5}\left(1-\frac{q^{\prime \prime}}{m_{d}}\right)\right] \\
=\frac{1}{2}\left[\frac{q^{\prime \mu}}{m_{d}}+\frac{p^{\prime \mu}(p q)}{m_{b} m_{c} m_{d}}-\frac{q^{\prime \mu}\left(p p^{\prime}\right)}{m_{b} m_{c} m_{d}}+\frac{p^{\mu}\left(p^{\prime} q^{\prime}\right)}{m_{b} m_{c} m_{d}}+\frac{p^{\prime \mu}}{m_{c}}+\frac{p^{\mu}}{m_{b}}\right]
\end{gathered}
$$

and

$$
\begin{gathered}
\overrightarrow{q^{\prime}}=-\vec{p}=-\overrightarrow{p^{\prime}}+\frac{\vec{P}_{D}}{M_{D}}\left(p_{D \|}\right) T_{D} \\
\phi_{B}=\frac{1}{\pi^{3 / 4} \beta_{B}^{3 / 2}} \exp \left[\frac{1}{2 \beta_{B}^{2}}\left(m_{b}^{2}-\left(p_{B \|}\right)^{2}\right)\right]=\frac{1}{\pi^{3 / 4} \beta_{B}^{3 / 2}} \exp \left[\frac{-\vec{p}^{2}}{2 \beta_{B}^{2}}\right] \\
\phi_{D}=\frac{1}{\pi^{3 / 4} \beta_{D}^{3 / 2}} \exp \left[\frac{1}{2 \beta_{D}^{2}}\left(m_{c}^{2}-\left(p_{D \|}\right)^{2}\right)\right]
\end{gathered}
$$

The trace which determines $\left\{V^{\mu}\right\}$ is analogous to formula (22) of Ref. [4] and the $\not q^{\prime} / m_{d}$ term can be connected with the momentum $\tilde{k}$ of the same reference. That term contains the Wigner rotation of the light quark. Comparison with (3.1) allows the extraction of the form factors, which can be computed numerically. In order to check Lorentz covariance, calculations leading to identical results have also been carried out in the D-meson rest frame $\left(\vec{P}_{D}=0\right)$. 
The decays with the vector meson $D^{*+}$ in the final states are described by expressions analogous to (3.3). The factor $\left\{V^{\mu}\right\}$ has to be replaced as follows:

$$
\left\{V^{\mu}\right\} \rightarrow\left\{V^{* \mu}\right\} \text { or }\left\{A^{* \mu}\right\}
$$

Here

$$
\begin{gathered}
\left\{V^{* \mu}\right\}=\operatorname{Tr}\left[\notin^{*} \frac{\not p^{\prime}+m_{c}}{2 m_{c}} \gamma^{\mu} \frac{\not p+m_{b}}{2 m_{b}} \gamma_{5} \frac{\not{ }^{\prime}-m_{d}}{2 m_{d}}\right] \\
\left\{A^{* \mu}\right\}=\operatorname{Tr}\left[\notin^{*} \frac{\not p^{\prime}+m_{c}}{2 m_{c}} \gamma^{\mu} \gamma_{5} \frac{\not p+m_{b}}{2 m_{b}} \gamma_{5} \frac{\not q^{\prime}-m_{d}}{2 m_{d}}\right]
\end{gathered}
$$

In the heavy-quark limit (HQL), one is tempted to identify the heavy-quark momenta with the heavy-meson momenta, for example,

$$
\overrightarrow{p^{\prime}}=\frac{m_{c}}{M_{D}} \vec{P}_{D}
$$

The Dirac delta-function constraints then determine

$$
\overrightarrow{q^{\prime}}=\frac{m_{d}}{M_{D}} \vec{P}_{D}
$$

This means that both valence quarks seem to travel as free particles. Indeed, with (3.9), one finds that

$$
m_{c}^{2}-\left(p_{D \|}\right)^{2}=0 \quad ; \quad \phi_{D}=\frac{1}{\pi^{3 / 4} \beta^{3 / 2}}
$$

One has failed to account for the Wigner rotation of the light quark [4] and all information on the internal quark momenta is lost. Thus, a more reasonable choice is

$$
\overrightarrow{p^{\prime}} \rightarrow \frac{m_{c}}{M_{D}} \vec{P}_{D}+\overrightarrow{k^{\prime}}, \quad\left|\overrightarrow{k^{\prime}}\right| / m_{c}<<1
$$

It leads to 


$$
\begin{gathered}
\phi_{D_{H Q L}}=\left.\frac{1}{\pi^{3 / 4} \beta^{3 / 2}} \exp \left[\frac{1}{2 \beta^{2}}\left(m_{c}^{2}-\left(p_{D \|}\right)^{2}\right)\right]\right|_{H Q L} \\
=\frac{1}{\pi^{3 / 4} \beta_{H Q L}^{3 / 2}} \exp \left[-\frac{1}{2 \beta_{H Q L}^{2}}\left({\overrightarrow{k^{\prime}}}^{2}-\frac{\left(\vec{P}_{D} \cdot \vec{k}^{\prime}\right)^{2}}{E_{D}^{2}}\right)\right] \\
\left(p_{D \|}\right)_{H Q L}^{2}=m_{c}^{2}+{\overrightarrow{k^{\prime}}}^{2}-\frac{\left(\overrightarrow{P_{D}} \cdot \overrightarrow{k^{\prime}}\right)^{2}}{E_{D}^{2}}+O\left(\frac{1}{m_{c}}\right)
\end{gathered}
$$

Furthermore, in the HQL, $m_{c} \rightarrow M_{D}$, so that

$$
\overrightarrow{k^{\prime}} \rightarrow \overrightarrow{p^{\prime}}-\vec{P}_{D}
$$

An analogous procedure is carried out for the B meson.

If one had chosen (3.9) instead of (3.12), one would have obtained

$$
\begin{gathered}
\left\{V^{\mu}\right\}=\frac{1}{4} \operatorname{Tr}\left[\gamma_{5}\left(1+\not \not^{\prime}\right) \gamma^{\mu}(1+\not \varnothing) \gamma_{5}\right] \\
\not \not^{\prime}=\frac{\not{ }^{\prime}}{m_{c}} \quad ; \quad \not 6=\frac{\not p}{m_{b}}
\end{gathered}
$$

Here the Wigner rotation of the light quark is absent. The expression (3.15) is analogous to the expressions employed by Ref. [5]. However, this reference does keep some information on the internal quark momenta in the valence-quark wave function, by retaining some relativistic terms [5], and thus evades the unacceptable result (3.11).

Finally, one finds, for example,

$$
\begin{gathered}
f_{+}\left(\vec{P}_{B}=0\right)_{H Q L}=\int d^{3} p^{\prime} I\left(p^{\prime}\right) \frac{M_{B}+M_{D}}{4 M_{B} M_{D}}\left[1+\frac{\tilde{\epsilon^{\prime}}}{m_{d}}-\frac{\left(\vec{P}_{D} \cdot \overrightarrow{\tilde{q}^{\prime}}\right)}{\left(E_{D}+M_{D}\right) m_{d}}\right] \\
I\left(p^{\prime}\right)=3(2 \pi)^{6} N_{D_{H Q L}}\left(\vec{P}_{D}\right) N_{B_{H Q L}}(0)\left(\frac{M_{D}}{E_{D}}\right)^{2} \frac{m_{d} M_{D}}{E_{D} \tilde{\epsilon}^{\prime}-\vec{P}_{D} \phi_{\bar{q}^{\prime}}} \phi_{D_{H Q L}} \phi_{B_{H Q L}}
\end{gathered}
$$


Here we have used

$$
\begin{aligned}
& \frac{e}{m_{b}} \rightarrow 1 \quad ; \quad \frac{e^{\prime}}{m_{c}} \rightarrow \frac{E_{D}}{M_{D}} \\
& \overrightarrow{g^{\prime}} \rightarrow \overrightarrow{\tilde{q}^{\prime}} \quad ; \quad \frac{\epsilon^{\prime}}{m_{d}} \rightarrow \frac{\tilde{\epsilon^{\prime}}}{m_{d}} \quad ; \quad \tilde{\epsilon^{\prime}}=\sqrt{m_{d}^{2}+\overrightarrow{\tilde{q}}^{\prime}} \\
& \overrightarrow{q^{\prime}}=-\overrightarrow{k^{\prime}}+\frac{\vec{P}_{D}}{M_{D}} \sqrt{m_{d}^{2}+\left({\overrightarrow{k^{\prime}}}^{2}-\frac{\left(\vec{P}_{D} \overrightarrow{k^{\prime}}\right)^{2}}{E_{D}^{2}}\right)} \\
& \frac{\overrightarrow{g^{\prime}}}{m_{b}} \rightarrow 0 \quad ; \quad \frac{\overrightarrow{p^{\prime}}}{m_{c}} \rightarrow \frac{\overrightarrow{P_{D}}}{M_{D}} \quad ; \quad \overrightarrow{k^{\prime}} \rightarrow \overrightarrow{p^{\prime}}-\overrightarrow{P_{D}} \\
& \phi_{B} \rightarrow \phi_{B_{H Q L}}=\frac{1}{\pi^{3 / 4} \beta_{H Q L}^{3 / 2}} \exp \left[\frac{{\overrightarrow{q^{\prime}}}^{2}}{2 \beta_{H Q L}^{2}}\right]
\end{aligned}
$$

Similar expressions are readily obtained for other form factors. Defining

$$
F_{1}=f_{+} \quad ; \quad A_{1}=\frac{1}{M_{B}+M_{D}} f ; \quad V=\left(M_{B}+M_{D}\right) g ; \quad A_{2}=-\left(M_{B}+M_{D}\right) a_{+}
$$

we find the well-known HQS relations [7]

$$
F_{1}=V=A_{2}=\frac{1}{1-\frac{Q^{2}}{\left(M_{B}+M_{D}\right)^{2}}} A_{1}
$$

This immediately shows that the definition (3.12) has not introduced any $\lambda / m_{Q}$ corrections. It only retained internal quark momenta and the Wigner rotation, which is necessary if inconsistencies and contradictions are to be avoided [4]. The relations (3.19) are valid only in the HQL. Then all form factors contain the same Isgur-Wise function (IWF), which is determined by

$$
\xi(w)=R f_{+}=R F_{1}
$$




$$
R=\frac{2 \sqrt{M_{B} M_{D}}}{M_{B}+M_{D}}
$$

Here

$$
w=v \cdot v^{\prime}=\frac{\left(P_{D} \cdot P_{B}\right)}{M_{D} M_{B}}
$$

In the B-meson rest frame $\left(\vec{P}_{B}=0\right)$ one finds

$$
\frac{E_{D}}{M_{D}}=w \quad ; \quad \frac{\left|\vec{P}_{D}\right|}{M_{D}}=\sqrt{w^{2}-1}
$$

The expression (3.20) also satisfies the well-known [7] constraint $\xi(1)=1$. In Fig.1 our IWF is compared with that calculated by Amundson [5], who obtained

$$
\xi(w)_{A}=\exp \left[-\frac{m_{d}^{2}}{2 \kappa^{2} \beta_{H Q L}^{2}}(w-1)\right]
$$

Our curve (solid line in Fig.1) is calculated using the parameters of Ref. [5]

$$
\beta_{H Q L}=0.42 \mathrm{GeV} \quad ; \quad m_{d}=0.33 \mathrm{GeV}
$$

A meson decay constant $f_{H}$ is determined by the expression

$$
\begin{gathered}
\frac{1}{(2 \pi)^{\frac{3}{2}}} P^{\mu} f_{H}=<0\left|: \bar{\psi}_{d}(0) \gamma^{\mu} \gamma_{5} \psi_{Q}(0):\right| H(E, \vec{P}, M)> \\
=\left.3 N(\vec{P}) \int d^{3} p \frac{m_{Q} M}{e E} \frac{M m_{d}}{E \epsilon-\vec{P} \vec{g}} \phi\left(l_{\perp}\right)\left[\frac{m_{d} p^{\mu}+m_{Q} q^{\mu}}{m_{d} m_{Q}}\right]\right|_{\vec{q}=-\vec{p}+\frac{\vec{P}}{M}\left(p_{\|}\right) T}
\end{gathered}
$$

Here $\psi_{Q}(x)$ are valence quark fields [1]. The decay constant $f_{H}$ is easily calculated in the frame $\vec{P}=0$. In order to check covariance numerically, it has also been calculated for several different $\vec{P}$ values. The results have always been identical.

With the parameters $[1,5]$

$$
\begin{array}{ll}
\beta_{S_{(u \bar{c})}}=0.39 \mathrm{GeV} & m_{u}=m_{d}=0.33 \mathrm{GeV} \\
\beta_{S_{(u \bar{b})}}=0.42 \mathrm{GeV} & m_{c}=1.645 \mathrm{GeV} \\
m_{b}=4.983 \mathrm{GeV}
\end{array}
$$


one finds that

$$
f_{D}=258,8 \mathrm{MeV} \quad ; \quad f_{B}=151,9 \mathrm{MeV}
$$

In the HQL, the expression (3.24) takes the form

$$
\frac{1}{(2 \pi)^{\frac{3}{2}}} P^{\mu} f_{H_{H Q L}}=3 N_{H Q L}(\vec{P}) \int d^{3} p\left(\frac{M}{E}\right)^{2} \frac{M m_{d}}{E \tilde{\epsilon}-\vec{P} \overrightarrow{\tilde{q}}} \phi_{H Q L}\left(l_{\perp}\right)\left[\frac{m_{d} P^{\mu}+M \tilde{q}^{\mu}}{M m_{d}}\right]
$$

In the HQL, one uses the average meson masses

$$
\bar{M}_{D}=\frac{3 M_{D^{*}}+M_{D}}{4}=1.975 \mathrm{GeV} \quad ; \quad \bar{M}_{B}=\frac{3 M_{B^{*}}+M_{B}}{4}=5.313 \mathrm{GeV}
$$

and (3.23) in order to find the HQS result

$$
f_{B_{H Q L}}=\sqrt{\frac{\bar{M}_{D}}{\bar{M}_{B}}} f_{D_{H Q L}}
$$

The numerical values

$$
f_{D_{H Q L}}=235,8 \mathrm{MeV} \quad ; \quad f_{B_{H Q L}}=143,8 \mathrm{MeV}
$$

which have been obtained using (3.27), are quite close to the result (3.26), showing that the model-determined corrections to the HQL are about $5 \div 6 \%$. 


\section{Gaussian sea}

It might be useful to demonstrate the flexibility of the expression (2.1) by selecting a sea function $\mathrm{F}(\mathrm{K})$ that would be different from the naive choice (2.4). In principle, this could be based on some QCD modelling of the sea contribution. However, for illustrative purposes a simple example can be selected, which in the NRL and WBL goes into the ISGW state. Yet, it leads to noticeably different results when used in the CQM. This is

$$
F(K)=\delta^{(4)}\left[K^{\mu}-\frac{P}{M}^{\mu}\left(\frac{P}{M}^{\nu}(P-(p+q))_{\nu}\right)\right] \cdot e^{-\alpha K^{2}}
$$

A simple arbitrary choice for the parameter $\alpha$ is

$$
\alpha=2^{-1} \beta^{-2}
$$

The integration over the sea momentum $\mathrm{K}$ gives

$$
K^{\mu}=\frac{P^{\mu}}{M}\left[M-\frac{E(e+\epsilon)-\frac{\vec{P}^{2}}{M}\left(p_{\|}\right) T}{M}\right]
$$

In the meson rest frame $\left(P^{\mu}=(M, 0)\right)$, this goes into $(2.5)$. In the WBL, one finds that

$$
\vec{K}=0 \quad ; \quad K^{0} \cong M-\left(m_{d}+m_{Q}\right)
$$

Thus the $K^{2}$, dependence has disappeared and (2.1) in the NRL-WBL is again the ISGW state (2.16). This conclusion is valid for any meson frame, i.e., any $P^{\mu}$.

In the CQM, all manipulations are exactly analogous to those presented in the second section of this paper. In all formulas one has to make the substitution 


$$
\phi\left(l_{\perp}\right) \rightarrow \phi_{K}=\phi\left(l_{\perp}\right) e^{-\alpha K^{2}}
$$

In the HQL, one can use (4.3) and (3.12)-(3.17) in order to obtain

$$
\begin{gathered}
K^{2}=\left(M-p_{\|}-q_{\|}\right)^{2} \rightarrow \tilde{q}_{\|}^{2} \\
\left(\phi_{K}\right)_{H Q L} \rightarrow \phi\left(l_{\perp}\right)_{H Q L} e^{-\alpha \tilde{q}_{\|}^{2}}
\end{gathered}
$$

Again, all formal deductions (3.17) are repeated with insertions of the factors $\exp \left[-\alpha \tilde{q}_{\|}^{2}\right]$ associated with the product $\phi_{D_{H Q L}} \cdot \phi_{B_{H Q L}}$. In the frame $\vec{P}_{B}=0$, for example, one has

$$
\phi_{D_{H Q L}} \phi_{B_{H Q L}} \rightarrow \phi_{D_{H Q L}} \phi_{B_{H Q L}} \cdot \exp \left\{-\alpha\left[\left(\tilde{q}_{D \|}\right)^{2}+\tilde{\epsilon}^{2}\right]\right\}
$$




\section{Numerical examples and discussion}

It is well known that various relativistic wave functions (states) can lead to the same state in the NRL. This has been illustrated in Secs. II and IV for two slightly different versions of the CQM. However, the different versions of the CQM lead to somewhat different estimates of physical quantities. These differences persist even in the HQL.

Owing to the relations (2.4), (4.1), or similar, the interplay of the sea and the valence-quark contribution leads to a meson mass $M$ which is not just a sum of the valence-quark masses. However, the values of the quark masses are interconnected with the quark wave function (2.3). For any mass change, the variational procedure which leads to (2.3) has to be repeated. Or alternatively, the meson wave function $\phi$ can be determined in some other model, for example, in a model inspired by the Bethe-Salpeter equation. For illustrative purposes, the mass $m_{d}$ has been changed in the model versions

determined by (2.3) and (2.4) or (4.1). Such a procedure, admittedly inconsistent, has been used just to illustrate the flexibility of the CQM's.

Table I

In Table I the meson decay form factors are calculated using (2.4) and the parameters $(3.23),(3.25)$, and (3.28). The spectator antiquark mass $m_{d}$ has been arbitrarily changed, as discussed above. As expected, the $f_{H}$ values change with $m_{d}$, but not dramatically, mostly by less than 20\%. Deviations from the HQL relation (3.29) are more interesting. They amount to abouth $9 \%$ when the full CQM is employed. This indicates that, in this version of the model, the HQS relation (3.29) presents a very good approximation. Absolute values $f_{B}(C Q M)$ are closer (less than $5 \%$ difference) to $f_{B_{H Q L}}$, as it should be expected with $m_{b} / m_{c}=3.3$.

\section{Table II}


The results presented in Table II show that the sea contributions can be very important. Whereas the general pattern is similar to that displayed in Table I, the absolute values of the meson decay constants $f_{H}$ are much smaller. For example, with $m_{d}=0.33 \mathrm{GeV}, f_{D}(4.1) / f_{D}(2.4)=0.59$, and $f_{B}(4.1) / f_{B}(2.4)=0.70$. However, this has to be taken more as an illustration of the model flexibility than as a serious prediction. The sea descriptions (2.4) or (4.1) are very crude and one should better refer to them as to "mock sea" functions.

Other calculations of the meson decay form factors lead to a broad range of values. A relativistic quark model with centrally confined quarks [8] gave values that were smaller up to $50 \%\left(f_{D}=130.6 \mathrm{MeV}, f_{B}=90.9 \mathrm{MeV}\right)$ than our (3.26) values or the values in Table I. These values are much closer to our values shown in Table II, which illustrates how model-based predictions depend on details of the model construction. The larger $f_{D}$ and $f_{B}$ values, as obtained in the present CQM, are closer to the results based on QCD sum rules, lattice calculations, and semilocal parton-hadron duality [9]. The estimates in the QCD sum rules [10] gave $f_{D}=120 \div 250 \mathrm{MeV}$ and $f_{B}=90 \div 200 \mathrm{MeV}$. The predictions of the lattice calculations [11] are in a similar range $f_{D}=170 \div 230 \mathrm{MeV}$ and $f_{B}=140 \div 220 \mathrm{MeV}$. Lower values, $f_{D} \cong 80 \mathrm{MeV}, f_{B} \cong 130 \mathrm{MeV}$, remarkably close to the values of Ref. [8], were found in a potential quark model [12].

The IWF calculated in the CQM defined by (2.4) (see Fig. 1) shows similar behavior to the ISGW-Amudson [5] result obtained with the correction factor $\kappa=0.6$. Its slope $\rho$ defined by

$$
\xi(w)=1-\rho^{2}(w-1)+O\left((w-1)^{2}\right)
$$

is about $25 \%$ larger than the result of Refs. [5]. This can be attributed to the fully relativistic character of the CQM, including Wigner rotation. As shown in Ref. [4] the Wigner rotation increases the slope of IWF by about $20 \%$.

Figure 2 shows the influence of the "mock sea" contribution. The solid curve and 
the dotted curve correspond to the sea functions (2.4) and (4.1), respectively. The use of (4.1) increases the slope $\rho$ by $10 \%$. One is tempted to assume that the relativistic effects (see Fig.1) might play a larger role in the calculation of the IWF than the sea effects. However, the model is too crude for such far-reaching conclusions.

An arbitrary d-antiquark mass change, using $m_{d}=0.1 \mathrm{GeV}$ instead of (3.23), produces virtually the same $\xi(w)$ curve with either the (2.4) or the (4.1) sea description. The slope $\rho=1.07$ is $9 \%$ smaller than the result based on (3.23) and (2.4) (see also Fig. 1).

A plethora of $\rho$ values can be found in the literature. By fitting the data on $B \rightarrow D l \nu$ and using different ansatze for the IWF [4,13-15], the $\rho$ values have been found to be in the range $\rho=0.92 \div 1.57$. This is slightly larger than the value based $[2,3,16-18]$ on ISGW model [1], $\rho \approx 0.8$. Relativistic quark models $[8,12]$ gave $\rho=1.25$ and $\rho=1.1$, respectively. The QCD sum-rule estimates $[19-26] \rho=1.0 \div 1.14$, as well as the lattice computations $[27-29] \rho=0.71 \div 1.35$, are more or less in the same range as the estimates [4,13-15] based on the $B \rightarrow D l \nu$ data. The values of the slope parameter $\rho$ shown in Figs. 1 and 2 , which roughly span the range $\rho=0.93 \div 1.29$, are similar to fitting-data estimates [4,13-15], or QCD sum-rule estimates [19-26], or lattice [27-29] estimates. The value obtained in the simplest CQM version of the ISGW model is $\rho=1.17$.

Our results obtained using (2.4) are connected with the Close and Wambach [4] deductions. In their case, the ISGW model was relativized sufficiently to make it covariant in the HQL. They have also taken care to include the Wigner rotation of light quarks. With $m_{Q}^{-1}$ corrections, their approach might also approximately, and adequatly, describe lighter mesons $(\mathrm{K})$.

When one starts with a covariant description, the HQL follows automatically by the $m_{Q}^{-1}$ expansion. The HQS results are readily obtained in this limit. The Wigner rotation is also automatically included in a covariant procedure. Furthermore, the CQM can be used for the description of the light mesons or the heavy-light meson transitions. 
A very general structure of the model, including the sea function $F(K)$, provides for its great adaptability and ability to model various physical situations. 


\section{References}

[1] N. Isgur, D. Scora, B. Grinstein, and M.B. Wise, Phys. Rev. D39, 799 (1989).

[2] N. Isgur, Phys. Rev. D43, 810 (1991).

[3] C. O. Dib and F. Vera, Phys. Rev. D47, 3938 (1993).

[4] F. E. Close and A. Wambach, Nucl. Phys. B412, 169 (1994).

[5] J. F. Amundson, Phys. Rev. D49, 373 (1994).

[6] L. Micu, Czechoslovak Journal of Physics 41, 129 (1991).

[7] M. Neubert, Phys. Rep. 245, 259 (1994).

[8] D. Tadić and S. Žganec, Phys. Rev. D50, 5853 (1994).

[9] S. Narison and K. Zalewski, Phys. Lett. B320, 369 (1994), and references therein.

[10] C. A. Dominguez, SISSA 139/39/EP, hep-th 9309260, presented at the 3rd Workshop on the $\tau$ /Charm Factory, Marbella, Spain, June 1993.

[11] C. T. Sachrajda, Talk given at the International Europhysics Conference in High Energy Physics, Marseille, France (1993); C. Bernard et al., (MILK), Preprint Wash. Univ. HEP/94-37, hep-lat 9411080.

[12] V. V. Kiselev, Preprint IHEP 94-77, September 1994.

[13] M. Neubert and V. Rieckert, Nucl. Phys. B382, 97 (1992).

[14] M. Neubert, Phys. Lett. B264, 445 (1991).

[15] J. L. Rosner, Phys. Rev. D42, 3732 (1990).

[16] E. de Rafael and J. Taron, Phys. Lett. B282, 215 (1992).

[17] M. A. Ivanov et al., Phys. Rev. D46, 3817 (1992). 
[18] E. Jenkins and M. J. Savage, Phys. Lett. B281, 331 (1992).

[19] A. A. Ovchinnikov and V. A. Slobodenyuk, Z. Phys. C44, 433 (1989).

[20] V. N. Baier and A. G. Grozin, Z. Phys. C47, 669 (1990).

[21] P. Ball, V. M. Braun, and H. G. Dosch, Phys. Rev. D44, 3567 (1991).

[22] A. F. Falk, H. Georgi, B. Grinstein, and M. B. Wise, Nucl. Phys. B343, 1 (1990).

[23] A. V. Radyushkin, Phys. Lett. B271, 218 (1991).

[24] M. Neubert, V. Rieckert, B. Stech, and Q. P. Xu, Heidelberg Preprint HD-THEP91-28, 1991.

[25] E. Bagan, P. Ball, V. M. Braun, and H. G. Dosch, Phys. Lett. B278, 457 (1992).

[26] P. Ball, Phys. Lett. B281, 133 (1992).

[27] C. Bernard, Y. Shen, and A. Soni, Phys. Lett. B317, 164 (1993).

[28] J. E. Mandula and M. C. Ogilvie, A Lattice Calculation of the Heavy Quark Universal Form Factors, hep-lat 9312013 (1993).

[29] S. P. Booth et al., Phys. Rev. Lett. 72, 462 (1994). 
Table I. Meson decay constants corresponding to formula (2.4).

\begin{tabular}{|c|c|c|c|c|}
\hline$m_{d}(\mathrm{GeV})$ & $f_{D}(\mathrm{MeV})$ & $f_{D_{H Q L}}(\mathrm{MeV})$ & $f_{B}(\mathrm{MeV})$ & $f_{B_{H Q L}}(\mathrm{MeV})$ \\
\hline 0.33 & 258.8 & 235.8 & 151.9 & 143.8 \\
0.3 & 251.4 & 233.2 & 149.3 & 142.2 \\
0.2 & 227.1 & 223.6 & 140.3 & 136.3 \\
0.1 & 204.3 & 212.6 & 131.2 & 129.6 \\
0.01 & 187.1 & 202.2 & 123.6 & 123.3 \\
\hline
\end{tabular}


Table II. Meson decay constants corresponding to formula (4.1).

\begin{tabular}{|c|c|c|c|c|}
\hline$m_{d}(\mathrm{GeV})$ & $f_{D}(\mathrm{MeV})$ & $f_{D_{H Q L}}(\mathrm{MeV})$ & $f_{B}(\mathrm{MeV})$ & $f_{B_{H Q L}}(\mathrm{MeV})$ \\
\hline 0.33 & 157.4 & 146.2 & 105.7 & 89.1 \\
0.3 & 153.8 & 144.5 & 104.4 & 88.1 \\
0.2 & 141.0 & 137.8 & 99.6 & 84.0 \\
0.1 & 127.2 & 129.2 & 93.5 & 78.8 \\
0.01 & 114.5 & 120.5 & 87.1 & 73.5 \\
\hline
\end{tabular}




\section{Figure captions}

Fig.1 The IWF's are shown as functions of $w$. The dashed curve shows the IWF calculated in Ref. [5] with $\kappa=1$. The dash-dotted curve shows the result of Ref. [5] with $\kappa=0.6$. The corresponding slope parameter is $\rho \approx 0.93$. The solid curve is

obtained using our formula (3.20). Its slope parameter $\rho \approx 1.17$ corresponds to the ansatz (5.1). All IWF's were calculated using the parameters (3.23).

Fig.2 Several IWF's are shown. The dashed curve, with $\rho \approx 1.07$, is obtained by using either (3.20) or (3.20) plus (4.7) substitution with $m_{d}=0.1 \mathrm{GeV}$. The solid curve, $\rho \approx 1.17$, is obtained using (3.20) and $m_{d}=0.33 \mathrm{GeV}$, the same as for the solid curve in Fig.1. The dotted curve is obtained with the Gaussian sea (4.7). Its slope parameter is $\rho \approx 1.29$. All slope parameters corresponds to the ansatz (5.1). 
This figure "fig1-1.png" is available in "png" format from: http://arxiv.org/ps/hep-ph/9509332v1 